\title{
Estimation of Partially Specified Spatial Autoregressive Model
}

\author{
Yuanqing ZHANG \\ College of Mathematics and Systems Science, Shandong University of Science and Technology, \\ Qingdao 266510, China; \\ School of Statistics and Management, Shanghai University of Finance and Economics, Shanghai \\ 200433, China \\ E-mail: zhangyuanqing980@sina.com
}

\begin{abstract}
In this paper, we study estimation of a partially specified spatial autoregressive model with heteroskedasticity error term. Under the assumption of exogenous regressors and exogenous spatial weighting matrix, we propose an instrumental variable estimation. Under some sufficient conditions, we show that the proposed estimator for the finite dimensional parameter is root- $n$ consistent and asymptotically normally distributed and the proposed estimator for the unknown function is consistent and also asymptotically distributed though at a rate slower than root- $n$. Monte Carlo simulations verify our theory and the results suggest that the proposed method has some practical value.
\end{abstract}

Keywords spatial; instrument variable; partially linear

\section{Introduction}

Since Paelinck coined the term "spatial econometrics" in the early 1970s to refer to a set of methods that explicitly handles with spatial dependence and spatial heterogeneity, this field has grown rapidly. The books by Cliff and Ord ${ }^{[1]}$, Anselin ${ }^{[2]}$, Cressie ${ }^{[3]}$, contribute significantly to the development of the field. For a recent survey about the subject, see Anselin and Bera ${ }^{[4]}$.

Among the class of spatial models, spatial autoregressive (SAR) models have attracted a lot of attention. Many methods have been proposed to estimate the SAR models, which include the method of maximum likelihood (ML) by $\mathrm{Ord}^{[5]}$ and Smirnov and Anselin ${ }^{[6]}$, the method of moments (MM) by Kelejian and Prucha ${ }^{[7-9]}$, and the method of quasi-maximum likelihood estimation (QMLE) by Lee ${ }^{[10]}$. A common characteristic of these methods is that they are all developed to estimate finite dimensional parameters in the SAR models which are assumed to be linear. Linearity is widely used in the parametric statistical inference and it needs many model assumptions. Although their properties are very well established, linear models are often unrealistic in applications. Moreover, mis-specification of the data generation by a linear model could lead to a large bias. To achieve greater realism, Su and Jin ${ }^{[1]}$ propose partially specified spatial autoregressive model in the following (hereafter PLSAR):

$$
y_{i}=\lambda_{0} \sum_{j=1}^{N} w_{i j} y_{j}+x_{i}^{\prime} \beta_{0}+g_{0}\left(z_{i}\right)+\varepsilon_{i}, i=1,2, \cdots, n
$$

Received November 12, 2013, accepted January 3, 2014

Supported by National Natural Science Foundation of China (Grant No. 71371118); the Graduate Innovation Fund Project of Shanghai University of Finance and Economics (Grant No. CXJJ-2011-444) 
where $y_{i}$ denotes the dependent variable of individual $i, x_{i}$ is $q$-dimensional explanatory variable and $z_{i}$ denotes $p$-dimensional explanatory variable, $w_{i j}$ denotes the spatial weight between individual $i$ and $j, \varepsilon_{i}$ denotes random noise, and $\lambda_{0}$ and $\beta_{0}$ denote the unknown true parameter value.

Recently, Su and Jin ${ }^{[11]}$ give a profiled quasi-maximum likelihood based estimator (PQMLE) and show that the rates of consistency for the finite dimensional parameters in the model depend on some general features of the spatial weight matrix. Unfortunately, A major weakness for $\mathrm{Su}$ and Jin ${ }^{[11]}$ 's PQMLE seems to be that the error term $\varepsilon_{i}$ 's in their model are required to be homoscedastically distributed. In the presence of heteroscedasticity, Lin and Lee ${ }^{[12]}$ have demonstrated that the QML-based estimator is usually inconsistent. Their estimator does not have a closed form expression. More recently, $\mathrm{Su}^{[13]}$ proposes semi-parametric GMM (SPGMM) estimation of semi-parametric SAR models where the spatial lag effect exists the model linearly and the exogenous variables enter the model nonparametrically, but the model of $\mathrm{Su}^{[13]}$ is absence of $x_{i}^{\prime} \beta$.

However, both estimators mentioned above seem to be computationally challenging for applied scientists and are not easy to use in practice. In this article, an alternative computationally simple estimator is proposed for consistently estimating PLSAR model (1) for both finite dimensional parameters and nonparametric component under heteroskedasticity in the model. We give the asymptotic normality and consistent covariance matrices for the parametric and nonparametric part. The estimators all have closed form expressions in the paper, so, it is easy to implement in practice. Monte Carlo simulation results indicate that our estimators perform well in finite samples.

The paper is organized as follows: Section 2 proposes an estimation of the model (1); Section 3 gives the asymptotic properties of the proposed estimators; Section 4 reports some Monte Carlo simulation results. All technical proofs are relegated to the appendices.

\section{Estimation}

There are two issues with estimation of the model (1). First is the correlation between the spatial term $\sum_{j=1}^{n} w_{i j} y_{j}$ and the error term $\varepsilon_{i}$ (i.e., endogeneity). Second is the infinite dimensionality of the unknown parameter $g_{0}(z)$, one can approximate it with a sieve method. Such an method was taken by Ai and Chen ${ }^{[14]}$ who show that the sieve estimator of the nonparametric component ( $g_{0}$ here) is consistent with a rate faster than $n^{-1 / 4}$ under certain metric; the estimator of the parametric component is $\sqrt{n}$ consistent and asymptotically normally distributed; the estimator for the asymptotic covariance of the parametric component estimator is consistent and easy to compute; and the optimally weighted minimum distance estimator of parametric component attains the semi-parametric efficiency bound. Specifically, let $p^{K}(z)=\left(p_{1}(z), p_{2}(z), \cdots, p_{K}(z)\right)^{\prime}$ denote a sieve estimator. For each $K$, there exists some constant $\pi_{0}$ such that $g_{0}(z)=p^{K}(z)^{\prime} \pi_{0}+v_{0}(z)$, with $v_{0}(z)$ satisfying $\left\|v_{0}(z)\right\| \rightarrow 0$ as $K \rightarrow \infty$.

Let $w_{i}=\left(w_{i 1}, w_{i 2}, \cdots, w_{i n}\right)^{\mathrm{T}}, Y=\left(y_{1}, y_{2}, \cdots, y_{n}\right)^{\mathrm{T}}$, We rewrite the model (1) yields

$$
y_{i}=\lambda w_{i}^{\mathrm{T}} Y+z_{i}^{\mathrm{T}} \beta+g\left(x_{i}\right)+\varepsilon_{i}
$$


Then taking conditional expectation yields

$$
E\left(y_{i} \mid x_{i}\right)=\lambda E\left(w_{i}^{\mathrm{T}} Y \mid x_{i}\right)+E\left(z_{i}^{\mathrm{T}} \mid x_{i}\right) \beta+g\left(x_{i}\right)+E\left(\varepsilon_{i} \mid x_{i}\right) .
$$

Since $E\left(\varepsilon_{i} \mid x_{i}\right)=0$, we get

$$
y_{i}-E\left(y_{i} \mid x_{i}\right)=\lambda\left\{w_{i}^{\mathrm{T}} Y-E\left(w_{i}^{\mathrm{T}} Y \mid x_{i}\right)\right\}+\left\{z_{i}^{\mathrm{T}}-E\left(z_{i}^{\mathrm{T}} \mid x_{i}\right)\right\} \beta+\varepsilon_{i}
$$

Finally, we deal with the endogeneity problem by applying the 2SLS. Let $h_{i}$ denote a column vector of instrumental variables. The instrumental variables include all exogenous regressors in the model. The instrument matrices $H$ have full column rank $r \geq q+1$.

Denote $p_{i}=p^{K}\left(z_{i}\right), P=\left(p_{1}, p_{2}, \cdots, p_{n}\right)^{\prime}, \delta_{0}=\left(\lambda_{0}, \beta_{0}^{\prime}\right)^{\prime}, \hat{y}_{i}=y_{i}-\hat{E}\left(y_{i} \mid x_{i}\right), \hat{Y}=$ $\left(\hat{y}_{1}, \hat{y}_{2}, \cdots, \hat{y}_{n}\right)^{\mathrm{T}} \hat{b}_{i}=\left(w_{i}^{\mathrm{T}} Y-\hat{E}\left(w_{i}^{\mathrm{T}} Y \mid x_{i}\right), z_{i}^{\mathrm{T}}-\hat{E}\left(z_{i}^{\mathrm{T}} \mid x_{i}\right)\right)^{\mathrm{T}}, \hat{B}=\left(\hat{b}_{1}, \hat{b}_{2}, \cdots, \hat{b}_{n}\right)^{\mathrm{T}}$, where

$$
\begin{gathered}
\hat{E}\left(z_{i} \mid x_{i}\right)=p^{K}\left(x_{i}\right)^{\mathrm{T}}\left(P^{\mathrm{T}} P\right)^{-1} \sum_{i=1}^{n} p^{K}\left(x_{i}\right)^{\mathrm{T}} z_{i}, \\
\hat{E}\left(y_{i} \mid x_{i}\right)=p^{K}\left(x_{i}\right)^{\mathrm{T}}\left(P^{\mathrm{T}} P\right)^{-1} \sum_{i=1}^{n} p^{K}\left(x_{i}\right)^{\mathrm{T}} y_{i}, \\
\hat{E}\left(w_{i}^{\mathrm{T}} Z \mid x_{i}\right)=p^{K}\left(x_{i}\right)^{\mathrm{T}}\left(P^{\mathrm{T}} P\right)^{-1} \sum_{i=1}^{n} p^{K}\left(x_{i}\right)^{\mathrm{T}} w_{i}^{\mathrm{T}} Z, \\
\hat{E}\left(w_{i}^{\mathrm{T}} Y \mid x_{i}\right)=p^{K}\left(x_{i}\right)^{\mathrm{T}}\left(P^{\mathrm{T}} P\right)^{-1} \sum_{i=1}^{n} p^{K}\left(x_{i}\right)^{\mathrm{T}} w_{i}^{\mathrm{T}} Y .
\end{gathered}
$$

The 2SLS estimators for the unknow parameters $\delta_{0}, \pi_{0}, g_{0}(z)$ are respectively given by

$$
\begin{aligned}
\hat{\delta} & =\left[\hat{B}^{\mathrm{T}} \hat{H}\left(\hat{H}^{\mathrm{T}} \hat{H}\right)^{-1} \hat{H}^{\mathrm{T}} \hat{B}\right]^{-1} \hat{B}^{\mathrm{T}} \hat{H}\left(\hat{H}^{\mathrm{T}} \hat{H}\right)^{-1} \hat{H}^{\mathrm{T}} \hat{Y} \\
\widehat{\pi} & =\left\{P^{\prime} P\right\}^{-1} P^{\prime}\{Y-B \widehat{\delta}\} \\
\widehat{g}(z) & =p^{K}(z)^{\prime} \widehat{\pi}
\end{aligned}
$$

\section{$3 \quad$ Asymptotic properties}

The following assumptions will be maintained throughout the paper.

Assumption 1 (i) $\left\{\left(X_{i}, Z_{i}, \varepsilon_{i}\right), i=1,2, \cdots, n\right\}$ are independently and identically distributed; (ii) for all $i, E\left[\varepsilon_{i} \mid X_{i}, Z_{i}, W\right]=0$; (iii) there exist some constants $\sigma_{\min }^{2}, \sigma_{\max }^{2}>0$ such that $\sigma_{\max }^{2} \geq \sigma_{i}^{2} \geq \sigma_{\min }^{2}$ holds, where $\sigma_{i}^{2}=E\left(\varepsilon_{i}^{2} \mid X_{i}, Z_{i}, W\right)$. (iv) for all $i, E\left[\varepsilon_{i}^{4} \mid X_{i}, Z_{i}, W\right]$ is finite and bounded.

Assumption 2 (i) The diagonal element of the spatial weighting matrix $W$ is zero; (ii) the matrix $I-\lambda_{0} W$ is nonsingular with $\left|\lambda_{0}\right|<1$, where $I$ is an $n \times n$ identity matrix; (iii) the row and column sums of the matrices $W,\left(I-\lambda_{0} W\right)^{-1}$ are bounded uniformly in absolute value.

Assumption 1 is needed for establishing the asymptotic distribution of the nonparametric estimator $\widehat{g}(z)$ and was used in [15]. Assumption 2 imposes restrictions on the spatial weighting matrix. These restrictions are commonly imposed in the spatial regression literature (e.g., Lee $\left.^{[16]}\right)$.

Assumption 3 For every $K$; (i) the smallest eigenvalue of $E\left[p_{i}^{\prime} p_{i}\right]$ is bounded away from zero uniformly in $K$ and (ii) there is a sequence of constants $\zeta_{0}(K)$ satisfying $\sup _{z \in \mathcal{Z}}\left\|p^{K}(z)\right\| \leq$ $\zeta_{0}(K)$, and $K=K(n)$ such that $\zeta_{0}(K)^{2} K / n \rightarrow 0$ as $n \rightarrow \infty$. 
Assumption 4 For any function $g(\cdot)$ satisfying the normalization of $g_{0}(\cdot)$, (i) there exist some $\alpha(>0), \pi=\pi(K), \sup _{z \in \mathcal{Z}}\left|g(z)-p^{K}(z)^{\prime} \pi\right|=O\left(K^{-\alpha}\right)$ as $K \rightarrow \infty$; (ii) $\sqrt{n} K^{-\alpha} \rightarrow 0$ and $n \rightarrow \infty$.

Assumption 5 The limits $Q_{\tilde{H}^{\mathrm{T}} \tilde{B}}=p \lim _{n \rightarrow \infty} n^{-1} \tilde{H}^{\mathrm{T}} \tilde{B}, Q_{\tilde{H}^{\mathrm{T}} \tilde{H}}=p \lim _{n \rightarrow \infty} n^{-1} \tilde{H}^{\mathrm{T}} \tilde{H}, Q_{\tilde{H}^{\mathrm{T}} \Lambda \tilde{H}}=$ $p \lim _{n \rightarrow \infty} n^{-1} \tilde{H}^{\mathrm{T}} \Lambda \tilde{H}=p \lim _{n \rightarrow \infty} n^{-1} \sum_{i=1}^{n} \tilde{h}_{i} \tilde{h}_{i}^{\mathrm{T}} \sigma_{i}^{2}$. exist and nonsingular.

Assumption 3 are imposed on the sieve approximations. Since the constant is not identified, we must impose some normalization on $g_{0}(\cdot)$ such as $g_{0}\left(z_{0}\right)=0$ at some point $z_{0}$ so that $g_{0}(\cdot)$ can be identified. The basis functions $p^{K}(z)$ shall be constructed to satisfy this normalization. In addtion, the assumption imposes a normalization on the approximating functions, bounding the second moment matrix away from singularity, and restricting the magnitude of the series terms. This condition is needed to ensure the convergence in probability of the sample second moment matrix of the approximating functions to their expectations. Without loss of generality, we will assume $E\left[p_{i}^{\prime} p_{i}\right]=I$. Basis functions satisfying this condition can always through appropriate normalization. Assumption 5 is a stability condition; the quantities are used in the expression of asymptotic variance of the estimator.

Define

$$
\begin{aligned}
& B^{*}=\left(C\left(G_{0}+X \beta_{0}\right), X\right) ; \\
& \Gamma_{n}^{\prime}=B^{* \prime}(I-S) M(I-S) ; \\
& \Lambda_{n}=\operatorname{diag}\left\{\sigma_{1}^{2}, \sigma_{2}^{2}, \cdots, \sigma_{n}^{2}\right\} ; \\
& \Omega_{n}=\left(Q_{\tilde{H}^{\mathrm{T}} \tilde{B}}^{\mathrm{T}} Q_{\tilde{H}^{\mathrm{T}} \tilde{H}}^{-1} Q_{\tilde{H}^{\mathrm{T}} \tilde{B}}\right)^{-1} Q_{\tilde{H}^{\mathrm{T}} \tilde{B}}^{\mathrm{T}} Q_{\tilde{H}^{\mathrm{T}} \tilde{H}}^{-1} Q_{\tilde{H}^{\mathrm{T}} \Lambda \tilde{H}} Q_{\tilde{H}^{\mathrm{T}} \tilde{H}}^{-1} Q_{\tilde{H}^{\mathrm{T}} \tilde{B}}\left(Q_{\tilde{H}^{\mathrm{T}} \tilde{B}}^{\mathrm{T}} Q_{\tilde{H}^{\mathrm{T}} \tilde{H}}^{-1} Q_{\tilde{H}^{\mathrm{T}} \tilde{B}}\right)^{-1} .
\end{aligned}
$$

The following assumptions will be maintained throughout.

Theorem 1 Suppose assumptions 1 5 are satisfied, then we can establish

$$
\Omega_{n}^{-1 / 2}\left(\widehat{\delta}-\delta_{0}\right) \rightarrow N(0, I) \text { in distribution. }
$$

For any given value $z_{0}$, denote

$$
\sigma_{n}^{2}\left(z_{0}\right)=p^{K}\left(z_{0}\right)^{\prime}\left\{P^{\prime} P\right\}^{-1} P^{\prime} \Lambda_{n} P\left\{P^{\prime} P\right\}^{-1} p^{K}\left(z_{0}\right) .
$$

We can establish the following theorem regarding the nonparametric component.

Theorem 2 Suppose Assumptions $1 \sim 5$ are satisfied, then we can establish: (i) $\widehat{\pi}=\pi_{0}+$ $O_{p}\left(\sqrt{K / n}+K^{-\alpha}\right)$; (ii) $\sup _{z \in \mathcal{Z}}\left|\widehat{g} f(z)-g_{0}(z)\right|=O_{p}\left(\zeta_{0}(K)\left[\sqrt{K} / \sqrt{n}+K^{-\alpha}\right]\right)$; (iii) $E\left[\left|\widehat{g}(z)-g_{0}(z)\right|^{2}\right]=$ $O_{p}\left(\left[K / n+K^{-2 \alpha}\right]\right)$; (iv) $\frac{\widehat{g}\left(z_{0}\right)-g_{0}\left(z_{0}\right)}{\sigma_{n}\left(z_{0}\right)} \rightarrow N(0,1)$ in distribution.

We estimate the residuals by $\widehat{\varepsilon}_{i}=Y_{i}-B_{i} \widehat{\delta}-P_{i} \widehat{\pi}$ and estimate $\sigma_{i}^{2}$ by $\widehat{\sigma}_{i}^{2}=\left(Y_{i}-B_{i} \widehat{\delta}-P_{i} \widehat{\pi}\right)^{2}$.

\section{Monte Carlo simulation}

In this section, we conduct a monte carlo experiment to evaluate finite sample performance of the proposed method. The data generating process is given by

$$
y_{i}=\lambda_{0} \sum_{j=1}^{N} w_{i j} y_{j}+x_{i}^{\prime} \beta_{0}+g_{0}\left(z_{i}\right)+\varepsilon_{i}, i=1,2, \cdots, n
$$

where $z_{i}$ follows the uniform distribution on $[0,1], x_{i}$ follows the exponential distribution with parameter 3 , and the error term $\varepsilon_{i}$ is normally distributed with mean zero and variance $\sigma^{2}$. 
We take the spatial weights matrix as the spatial scenario in [17] with $R$ number of districts, $m$ members in each district, and with each neighbor of a member in a district given equal weight, i.e., $W=I_{R} \otimes B_{m}$, where $B_{m}=\left(l_{m} l_{m}^{\prime}-I_{m}\right) /(m-1), m=10$, where $l_{m}$ denotes $m$-dimensional column vector with all elements of $1, I_{m}$ denotes identity matrix. We consider $g(z)=6 \cos (2 \pi z), \lambda=-0.5,0$, or 0.5 , and $\beta=-6$ or 6 . In the simulations, we take $K=6$ and $p^{K}(z)=\left(1, z, z^{2}, z^{3}, z^{4}, z^{5}\right)^{\prime}$.

Table 1 summarizes the simulation results for estimating $\lambda, \beta$ and $g(z)$ under different selections of the true parameters for $n=200,500$, and 1000. Each entry is based on 1000 repetitions.

Table 1 when $\sigma^{2}=1$, Summary of Bias, SEE, ESE and CP for $\lambda$ and $\beta$, the estimator for $\sigma^{2}$ under model (8)

\begin{tabular}{|c|c|c|c|c|c|c|c|c|c|c|}
\hline \multirow[b]{2}{*}{$n$} & \multirow{2}{*}{$\begin{array}{l}\sigma^{2} \\
\hat{\sigma}^{2}\end{array}$} & \multicolumn{4}{|c|}{$\lambda$} & \multicolumn{4}{|c|}{$\beta$} & \multirow{2}{*}{$\begin{array}{c}g(z) \\
\text { RISE }\end{array}$} \\
\hline & & Bias & SEE & ESE & $\mathrm{CP}$ & Bias & SEE & $\mathrm{ESE}$ & $\mathrm{CP}$ & \\
\hline & & \multicolumn{4}{|c|}{$\lambda=-0.5$} & \multicolumn{4}{|c|}{$\beta=6$} & \\
\hline 200 & 0.9706 & 0.0092 & 0.0720 & 0.0742 & 0.946 & 0.0056 & 0.2129 & 0.2186 & 0.944 & 0.2466 \\
\hline 500 & 0.9952 & -0.0009 & 0.0434 & 0.0436 & 0.945 & 0.0050 & 0.1385 & 0.1371 & 0.943 & 0.1740 \\
\hline \multirow[t]{2}{*}{1000} & 1.0020 & 0.0008 & 0.0293 & 0.0301 & 0.960 & 0.0010 & 0.0963 & 0.0957 & 0.949 & 0.1458 \\
\hline & & \multicolumn{4}{|c|}{$\lambda=0$} & \multicolumn{4}{|c|}{$\beta=6$} & \\
\hline 200 & 0.9699 & 0.0072 & 0.0504 & 0.0501 & 0.948 & 0.0048 & 0.2119 & 0.2176 & 0.942 & 0.2457 \\
\hline 500 & 0.9951 & 0.0007 & 0.0306 & 0.0299 & 0.940 & 0.0013 & 0.1379 & 0.1367 & 0.943 & 0.1738 \\
\hline \multirow[t]{2}{*}{1000} & 1.0020 & -0.0006 & 0.0207 & 0.0207 & 0.955 & 0.0011 & 0.09611 & 0.0954 & 0.949 & 0.1456 \\
\hline & & \multicolumn{4}{|c|}{$\lambda=0.5$} & \multicolumn{4}{|c|}{$\beta=6$} & \\
\hline 200 & 0.9691 & 0.0042 & 0.0265 & 0.0265 & 0.951 & 0.0046 & 0.2123 & 0.2178 & 0.944 & 0.2456 \\
\hline 500 & 0.995 & 0.0004 & 0.0161 & 0.0160 & 0.942 & 0.0036 & 0.1381 & 0.137 & 0.942 & 0.1739 \\
\hline \multirow[t]{2}{*}{1000} & 1.0020 & -0.0003 & 0.0109 & 0.0112 & 0.957 & 0.0015 & 0.0964 & 0.0957 & 0.948 & 0.1456 \\
\hline & & \multicolumn{4}{|c|}{$\lambda=-0.5$} & \multicolumn{4}{|c|}{$\beta=-6$} & \\
\hline 200 & 0.9706 & 0.0086 & 0.0688 & 0.0736 & 0.944 & 0.0061 & 0.2121 & 0.2186 & 0.947 & 0.2446 \\
\hline 500 & 0.9952 & 0.0006 & 0.0421 & 0.0438 & 0.957 & 0.0023 & 0.1386 & 0.1372 & 0.943 & 0.1721 \\
\hline \multirow[t]{2}{*}{1000} & 1.0020 & -0.0005 & 0.0289 & 0.0300 & 0.954 & 0.0019 & 0.0963 & 0.0957 & 0.947 & 0.1453 \\
\hline & & \multicolumn{4}{|c|}{$\lambda=0$} & \multicolumn{4}{|c|}{$\beta=-6$} & \\
\hline 200 & 0.9699 & 0.0068 & 0.0482 & 0.0498 & 0.942 & 0.0060 & 0.2116 & 0.2177 & 0.944 & 0.2438 \\
\hline 500 & 0.9952 & 0.0005 & 0.0297 & 0.0300 & 0.956 & 0.0043 & 0.1384 & 0.1367 & 0.940 & 0.1720 \\
\hline \multirow[t]{2}{*}{1000} & 1.0020 & -0.0002 & 0.0204 & 0.0207 & 0.950 & 0.0021 & 0.0962 & 0.0954 & 0.948 & 0.1452 \\
\hline & & \multicolumn{4}{|c|}{$\lambda=0.5$} & \multicolumn{4}{|c|}{$\beta=-6$} & \\
\hline 200 & 0.9691 & 0.0040 & 0.0253 & 0.0264 & 0.951 & 0.0070 & 0.2123 & 0.2179 & 0.944 & 0.2438 \\
\hline 500 & 0.9952 & 0.0003 & 0.0157 & 0.0161 & 0.959 & 0.0061 & 0.1390 & 0.1369 & 0.938 & 0.1722 \\
\hline 1000 & 1.0020 & $-3.8 \mathrm{e}-05$ & 0.0108 & 0.0111 & 0.952 & 0.0023 & 0.0966 & 0.0957 & 0.952 & 0.1452 \\
\hline
\end{tabular}

For each estimator, Bias is the average of estimation biases from 1000 repetitions, SSE is the sample standard error of the estimates, ESE is the average of the estimated standard error, 
let SEE denote the sampling standard error of the estimates, CP is the coverage probability of a 95 percent confidence interval for the estimator, RISE is defined as the average of the square root of integrated square error of $\hat{g}(z)$ where for each repetition,

$$
\operatorname{RISE}(\hat{g}(z))=\sqrt{\int_{\mathcal{Z}}\{\hat{g}(z)-g(z)\}^{2} \mathrm{~d} F(z)},
$$

where $\mathcal{Z}$ is the support of $z, F(z)$ is the distribution function of $z$.

Table 1 shows that $\hat{\sigma}^{2}$ is close to the true value 1 as $n$ becomes larger. The biases of $\lambda$ and $\beta$ become smaller as $n$ becomes larger. The SEE and ESE for $\hat{\lambda}$ and $\hat{\beta}$ are very close. The coverage probabilities of $\hat{\lambda}$ and $\hat{\beta}$ are close to 0.95 nominal level. The RISE of $g(z)$ becomes smaller as $n$ becomes larger. Figure 1 shows the comparison of the true function of $g(z)$ and the estimating function of $\hat{g}(z)$.
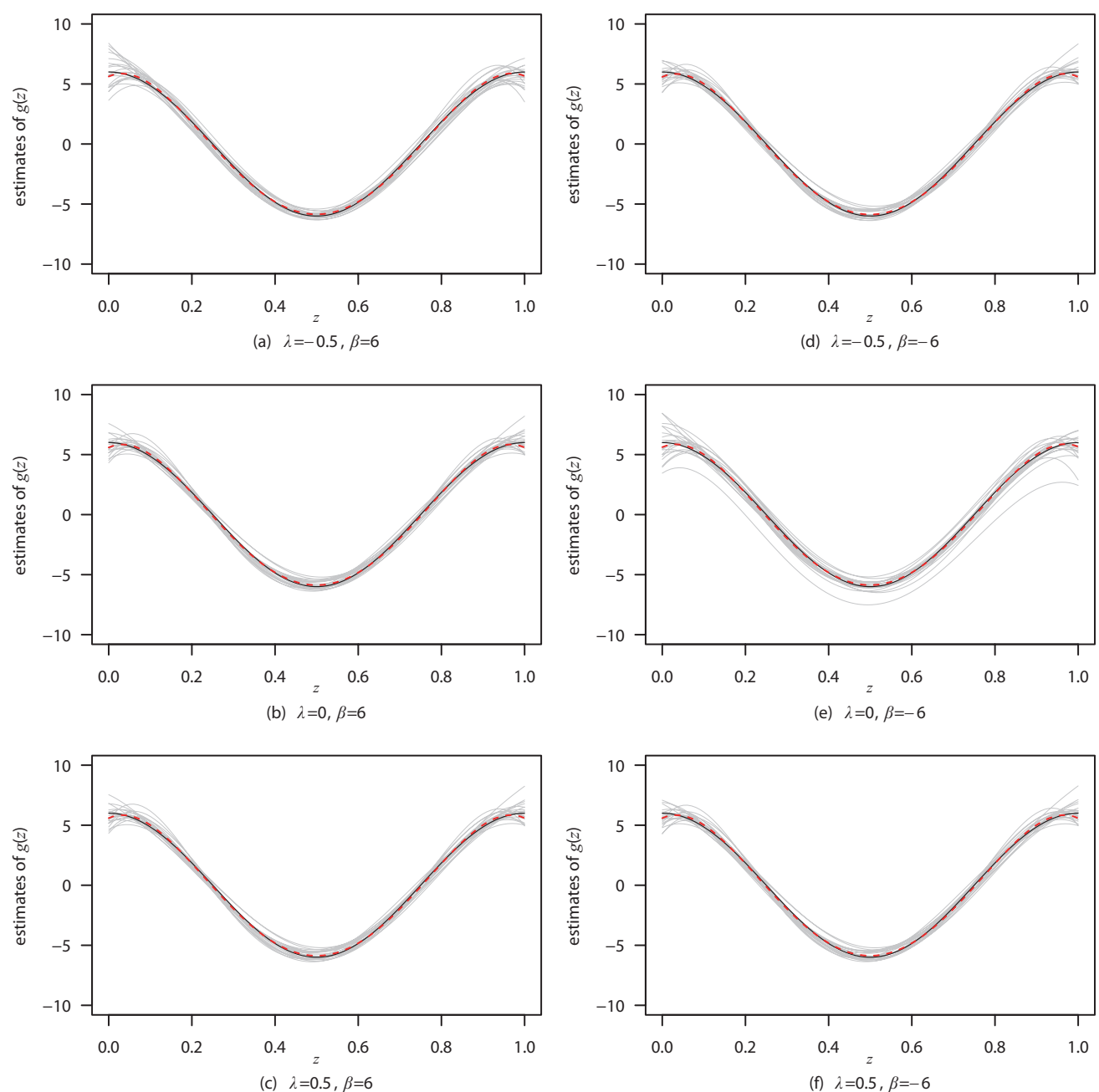

The black dashed line is the true function. The dotted red line is the average estimate of 1000 simulations. The grey lines are the estimated $g(z)$ in 20 simulations.

Figure 1 The plots of the estimates of $g(z)=6 \cos (2 \pi z)$ under model (8) with $n=200$ and $\sigma=1$ for different values of $\lambda$ and $\beta$. 


\section{Appendix technicalities}

We denote $\operatorname{tr}(A)=\operatorname{trace}(A)$ for a square matrix $A$.

Before we prove the main results, we first give some useful convergence results. By Assumption 3(i), we assume without loss of generality that: $E\left\{p_{k K}\left(z_{i}\right) p_{j K}\left(z_{i}\right)\right\}=I_{j k}$, where $I_{j k}$ denotes the $(j, k)$ th element of the identity matrix. Let $\|\cdot\|$ denotes the Euclidean norm. Denote $Q=\frac{P^{\prime} P}{n}$, we obtain

$$
\|Q-I\|=O_{p}\left(\zeta_{0}(K) \sqrt{K} / \sqrt{n}\right)=o_{p}(1)
$$

Let $1_{n}$ denote the indicator function for the smallest eigenvalue of $Q$ being greater than $1 / 2$. Then $\lim _{n \rightarrow \infty} P\left(1_{n}=1\right)=1$. By Assumption 1, we obtain

$$
1_{n}\left\|Q^{-1 / 2} P^{\prime} \varepsilon / n\right\|=O_{p}(\sqrt{K} / \sqrt{n})
$$

and

$$
\begin{gathered}
1_{n}\left\|Q^{-1} P^{\prime} \varepsilon / n\right\|=O_{p}(\sqrt{K} / \sqrt{n}) \\
1_{n}\left\|Q^{-1} P^{\prime} V_{0} / n\right\|=O_{p}\left(K^{-\alpha}\right)
\end{gathered}
$$

\section{Proof of Theorem 1}

For the sake of convenience, we take the instrument variables as $h_{i}=\left(x_{i} w_{i}^{\mathrm{T}} Z, \tilde{z}_{i}^{\mathrm{T}}\right)^{\mathrm{T}}$ in the proof, $H=\left(h_{1}, h_{2}, \cdots, h_{n}\right)^{\mathrm{T}} . m\left(u_{i}\right)=E\left[h_{i} \mid u_{i}\right], \nu_{i}=h_{i}-m\left(u_{i}\right)$, we will use the shorthand notation: $m_{i}=m\left(u_{i}\right)$, the variables without the subscript represent the matrices, e.g, $m=$ $\left(m_{1}, m_{2}, \cdots, m_{n}\right)^{\mathrm{T}}$. For any matrix $A$, we define $\vec{A}=P\left(P^{\mathrm{T}} P\right)^{-1} P^{\mathrm{T}} A$, applying this definition to $\tilde{Y}, \tilde{m}, \varepsilon, \tilde{\nu}$, we get $\vec{Y}, \vec{m}, \vec{\varepsilon}, \vec{\nu}$. From $h_{i}=m_{i}+\nu_{i}$, we get $\vec{h}_{i}=\vec{m}_{i}+\vec{\nu}_{i}$, or in vector matrix notation, $H=m+\nu, \vec{H}=\vec{m}+\vec{\nu}$. For scalars and vectors $A_{1 i}$ and $A_{2 i}$, let us define $S_{A 1, A 2}=$ $n^{-1} \sum_{i} A_{1 i} A_{2 i}^{\mathrm{T}}$, Also we let $S_{A_{1}, A_{1}}=S_{A_{1}}$ Because

$$
\begin{aligned}
E\left\|S_{\nu, \vec{\varepsilon}} \mid X\right\|^{2} & =n^{-2} \operatorname{tr}\left[P\left(P^{\mathrm{T}} P\right)^{-1} P^{\mathrm{T}} \nu \nu^{\mathrm{T}} P\left(P^{\mathrm{T}} P\right)^{-1} P^{\mathrm{T}} E\left(\varepsilon \varepsilon^{\mathrm{T}} \mid X\right)\right] \\
& \leq C n^{-2} \operatorname{tr}\left[P\left(P^{\mathrm{T}} P\right)^{-1} P^{\mathrm{T}} \nu \nu^{\mathrm{T}} P\left(P^{\mathrm{T}} P\right)^{-1} P^{\mathrm{T}}\right] \\
& \leq C n^{-2} \operatorname{tr}\left[\vec{\nu} \vec{\nu}^{\mathrm{T}}\right] \leq C n^{-1} S_{\vec{\nu}}=O\left(K / n^{2}\right) .
\end{aligned}
$$

Hence,

$$
S_{\nu, \vec{\varepsilon}}=O_{p}(\sqrt{K} / n)
$$

Furthermore,

$$
S_{m-\vec{m}, \vec{\varepsilon}} \leq\left\{S_{m-\vec{m}} S_{\vec{\varepsilon}}\right\}^{-1 / 2}=O_{p}\left(K^{-\alpha}\right) O_{p}(\sqrt{K} / \sqrt{n}),
$$

and

$$
S_{\vec{\nu}, \vec{\varepsilon}} \leq\left\{S_{\vec{\nu}} S_{\vec{\varepsilon}}\right\}^{-1 / 2}=O_{p}(K / n) .
$$

Because

$$
S_{H-\vec{H}, \vec{\varepsilon}}=S_{\nu+(m-\vec{m})-\vec{\nu}, \vec{\varepsilon}}=S_{\nu, \vec{\varepsilon}}+S_{m-\vec{m}, \vec{\varepsilon}}+S_{\vec{\nu}, \vec{\varepsilon}},
$$

So,

$$
S_{H-\vec{H}, \vec{\varepsilon}}=o_{p}\left(n^{-1 / 2}\right) .
$$

Because

$$
E\left\|S_{m-\vec{m}, \varepsilon} \mid X\right\|^{2}=n^{-2} \operatorname{tr}\left[(m-\vec{m})(m-\vec{m})^{\mathrm{T}} E\left(\varepsilon \varepsilon^{\mathrm{T}} \mid X\right)\right]
$$




$$
\leq C n^{-2} \operatorname{tr}\left[(m-\vec{m})(m-\vec{m})^{\mathrm{T}}\right] \leq C n^{-1} S_{m-\vec{m}}=o_{p}\left(n^{-1}\right),
$$

Hence,

$$
S_{m-\vec{m}, \varepsilon}=o_{p}\left(n^{-1 / 2}\right)
$$

Because

$$
E\left\|S_{\vec{\nu}, \varepsilon} \mid X\right\|^{2} \leq C n^{-1} \operatorname{tr}\left[S_{\vec{\nu}}\right]=o_{p}\left(n^{-1}\right)
$$

Hence,

$$
\begin{gathered}
S_{\vec{\nu}, \varepsilon}=o_{p}\left(n^{-1 / 2}\right) \\
S_{H-\vec{H}, \varepsilon}=S_{\nu+(m-\vec{m})-\vec{\nu}, \varepsilon}=S_{\nu, \varepsilon}+S_{m-\vec{m}, \varepsilon}-S_{\vec{\nu}, \varepsilon} . \\
\sqrt{n} S_{H-\vec{H}, \varepsilon}=\sqrt{n} S_{\nu, \varepsilon}+o_{p}(1) .
\end{gathered}
$$

So,

$$
\begin{gathered}
\sqrt{n} S_{H-\vec{H}, \varepsilon} \rightarrow N\left(0, Q_{\tilde{H}^{\mathrm{T}} \Lambda \tilde{H}}\right) . \\
{\left[\frac{\hat{B}^{\mathrm{T}} \hat{H}}{n}\left(\frac{\hat{H}^{\mathrm{T}} \hat{H}}{n}\right)^{-1} \frac{\hat{H}^{\mathrm{T}} \hat{B}}{n}\right]^{-1} \frac{\hat{B}^{\mathrm{T}} \hat{H}}{n}\left(\frac{\hat{H}^{\mathrm{T}} \hat{H}}{n}\right)^{-1}} \\
\rightarrow\left\{Q_{\tilde{H}^{\mathrm{T}} \tilde{B}}^{\mathrm{T}} Q_{\tilde{H}^{\mathrm{T}} \tilde{H}}^{-1} Q_{\tilde{H}^{\mathrm{T}} \tilde{B}}^{-1} Q_{\tilde{H}^{\mathrm{T}} \tilde{B}^{\mathrm{T}} Q_{\tilde{H}^{\mathrm{T}} \tilde{H}}^{-1}}\right. \\
\sqrt{n}[\hat{\delta}-\delta]=\left[\frac{\hat{B}^{\mathrm{T}} \hat{H}}{n}\left(\frac{\hat{H}^{\mathrm{T}} \hat{H}}{n}\right)^{-1} \frac{\hat{H}^{\mathrm{T}} \hat{B}}{n}\right]^{-1} \frac{\hat{B}^{\mathrm{T}} \hat{H}}{n}\left(\frac{\hat{H}^{\mathrm{T}} \hat{H}}{n}\right)^{-1} \sqrt{n}\left\{S_{H-\vec{H}, \varepsilon}-S_{H-\vec{H}, \vec{\varepsilon}}\right\}
\end{gathered}
$$

Thus,

$$
\sqrt{n}[\hat{\delta}-\delta]=\left\{Q_{\tilde{H}^{\prime} \tilde{B}}^{\mathrm{T}} Q_{\tilde{H}^{\mathrm{T}} \tilde{H}}^{-1} Q_{\tilde{H}^{\mathrm{T}} \tilde{B}}\right\}^{-1} Q_{\tilde{H}^{\mathrm{T}} \tilde{B}}^{\mathrm{T}} Q_{\tilde{H}^{\mathrm{T}} \tilde{H}^{-1}}^{-1} N\left(0, Q_{\tilde{H}^{\mathrm{T}} \Lambda \tilde{H}}\right)+o_{p}(1),
$$

The proof of Theorem is completed following an application of the Crémer-Wald device.

\section{Proof of Theorem 2}

We write

$$
\widehat{\pi}=\left\{P^{\prime} P\right\}^{-1} P^{\prime}\{Y-B \widehat{\delta}\}=\left\{P^{\prime} P\right\}^{-1} P^{\prime}\left\{Y-B \delta_{0}\right\}+\left\{P^{\prime} P\right\}^{-1} P^{\prime} B\left(\widehat{\delta}-\delta_{0}\right) .
$$

Consider the second term. We have

$$
\begin{aligned}
& \left\|\left(P^{\prime} P\right)^{-1} P^{\prime} B\left(\widehat{\delta}-\delta_{0}\right)\right\|^{2}=\left(\widehat{\delta}-\delta_{0}\right)^{\prime} \frac{B^{\prime} P}{n}\left(\frac{P^{\prime} P}{n}\right)^{-2} \frac{P^{\prime} B}{n}\left(\widehat{\delta}-\delta_{0}\right) \\
= & O_{p}\left(\frac{1}{n}\right)\left(\widehat{\delta}-\delta_{0}\right)^{\prime} B^{\prime} S B\left(\widehat{\delta}-\delta_{0}\right)=O_{p}\left(\frac{1}{n}\right) \text { by Assumption } 5 .
\end{aligned}
$$

Thus,

$$
\begin{aligned}
\widehat{\pi} & =\left\{P^{\prime} P\right\}^{-1} P^{\prime}\left\{Y-B \delta_{0}\right\}+O_{p}\left(n^{-1 / 2}\right) \\
& =\pi_{0}+\left\{P^{\prime} P\right\}^{-1} P^{\prime} \varepsilon+\left\{P^{\prime} P\right\}^{-1} P^{\prime} V_{0}+O_{p}\left(n^{-1 / 2}\right) \\
& =\pi_{0}+O_{p}\left(\sqrt{K / n}+K^{-\alpha}\right)
\end{aligned}
$$

by applying (11). Notice that

$$
\begin{gathered}
1_{n}\left|\hat{g}(z)-g_{0}(z)\right| \leq\left|p^{K}(z)^{\prime}\left(\hat{\pi}-\pi_{0}\right)\right|+\left|p^{K}(z)^{\prime} \pi_{0}-g_{0}(z)\right| \\
\leq \zeta_{0}(K) 1_{n}\left\|\hat{\pi}-\pi_{0}\right\|+O\left(K^{-\alpha}\right)=O_{p}\left(\zeta_{0}(K)\left[\sqrt{K} / \sqrt{n}+K^{-\alpha}\right]\right) .
\end{gathered}
$$


We obtain

$$
\hat{g}(z)=g_{0}(z)+O_{p}\left(\zeta_{0}(K)\left[\sqrt{K} / \sqrt{n}+K^{-\alpha}\right]\right)
$$

uniformly over $z$ and

$$
\sup _{z \in \mathcal{Z}}\left|\hat{g}(z)-g_{0}(z)\right|=O_{p}\left(\zeta_{0}(K)\left[\sqrt{K} / \sqrt{n}+K^{-\alpha}\right]\right) .
$$

Furthermore, applying (12) and Assumption 3(ii), we obtain

$$
\begin{aligned}
1_{n} E\left[\left(\hat{g}(z)-g_{0}(z)\right)^{2}\right] & =1_{n} E\left[p^{K}(z)^{\prime}\left(\hat{\pi}-\pi_{0}\right)+\left(p^{K}(z)^{\prime} \pi_{0}-g_{0}(z)\right)\right]^{2} \\
& \leq 2 * 1_{n}\left\|\hat{\pi}-\pi_{0}\right\|^{2}+2 * 1_{n} E\left[p^{K}(z)^{\prime} \pi_{0}-g_{0}(z)\right]^{2} \\
& =O_{p}\left(K / n+K^{-2 \alpha}\right)+O\left(K^{-2 \alpha}\right)=O_{p}\left(K / n+K^{-2 \alpha}\right) .
\end{aligned}
$$

Notice that

$$
p^{K}\left(z_{0}\right)^{\prime}\left(P^{\prime} P\right)^{-1} P^{\prime} B\left(\widehat{\delta}-\delta_{0}\right)=O_{p}\left(\zeta_{0}(K) n^{-1 / 2}\right) .
$$

By substitution and at a fixed $z_{0}$, we have

$$
\begin{aligned}
& \widehat{g}\left(z_{0}\right)-g_{0}\left(z_{0}\right)=p^{K}\left(z_{0}\right)^{\prime} \hat{\pi}-\left[p^{K}\left(z_{0}\right)^{\prime} \pi_{0}+O\left(K^{-\alpha}\right)\right] \\
= & p^{K}\left(z_{0}\right)^{\prime}\left[\pi_{0}+\left\{P^{\prime} P\right\}^{-1} P^{\prime} \varepsilon+\left\{P^{\prime} P\right\}^{-1} P^{\prime} V_{0}+O_{p}\left(n^{-1 / 2}\right)\right]-\left[p^{K}\left(z_{0}\right)^{\prime} \pi_{0}+O\left(K^{-\alpha}\right)\right] \\
= & p^{K}\left(z_{0}\right)^{\prime}\left\{P^{\prime} P\right\}^{-1} P^{\prime} \varepsilon+O_{p}\left(\zeta_{0}(K)\left[n^{-1 / 2}+K^{-\alpha}\right]\right) .
\end{aligned}
$$

Let $\xi_{\text {in }}=\frac{p^{K}\left(z_{0}\right)^{\prime}\left\{P^{\prime} P\right\}^{-1} P_{i} \varepsilon_{i}}{\sigma_{n}\left(z_{0}\right)}$, note that for each $n, E\left[\xi_{i n}\right]=0, s_{n}^{2}=\sum_{i=1}^{n} E\left[\xi_{i n}^{2}\right]=1$.

For every $\epsilon>0$,

$$
\begin{aligned}
& \frac{1}{s_{n}^{2}} \sum_{i=1}^{n} E\left[1\left(\left|\xi_{\text {in }}\right|>\epsilon s_{n}\right) \xi_{\text {in }}^{2}\right]=\sum_{i=1}^{n} \epsilon E\left[1\left(\left|\xi_{\text {in }} / \epsilon\right|>1\right) \xi_{\text {in }}^{2}\right] \\
= & \sum_{i=1}^{n} \epsilon^{2} E\left[1\left(\left|\xi_{\text {in }} / \epsilon\right|>1\right)\left(\xi_{\text {in }} / \epsilon\right)^{2}\right] \leq \sum_{i=1}^{n} \epsilon^{2} E\left[\left(\xi_{\text {in }} / \epsilon\right)^{4}\right] \\
\leq & \sum_{i=1}^{n} \epsilon^{2}\left\|\frac{p^{K}\left(z_{0}\right)^{\prime}}{\sigma_{n}\left(z_{0}\right)}\right\|^{4} \times\left\|\left(P^{\mathrm{T}} P / n\right)^{-1}\right\|^{4} \zeta_{0}(K)^{2} E\left\{\left\|P_{i}\right\|^{2} E\left[\varepsilon_{i}^{4} \mid Z\right]\right\} / n^{2} \epsilon^{4} \\
\leq & C \zeta_{0}(K)^{2} K / n \rightarrow 0 \text { by Assumptions } 2,3,4 .
\end{aligned}
$$

where $1(\cdot)$ denotes indicative function. Then, by the Lindbergh-Feller central limit theorem, $\sum_{i=1}^{n} \xi_{\text {in }} \rightarrow N(0,1)$ in distribution. So,

$$
\frac{p^{K}\left(z_{0}\right)^{\prime}\left\{P^{\prime} P\right\}^{-1} P^{\prime} \varepsilon}{\sigma_{n}\left(z_{0}\right)} \rightarrow N(0,1) \text { in distribution. }
$$

Thus,

$$
\frac{\widehat{g}(z)-g_{0}(z)}{\sigma_{n}\left(z_{0}\right)} \rightarrow N(0,1) \text { in distribution. }
$$

Acknowledgements We thank the referees for their time and comments.

\section{References}

[1] Cliff A D, Ord J K. Spatial autocorrelation. London: Pion Ltd, 1973.

[2] Anselin L. Spatial econometrics: Methods and models. The Netherlands: Kluwer Academic Publishers, 1988. 
[3] Cressie N. Statistics for spatial data. JohnWiley Sons, New York, 1993.

[4] Anselin L, Bera A K. Spatial dependence in linear regressionmodels with an introduction to spatial econometrics. In: Ullah A, Giles D E A. Handbook of Applied Economics Statistics. Marcel Dekker, New York, 2002.

[5] Ord J K. Estimation methods for models of spatial interaction. Journal of the American Statistical Association, 1975, 70: 120-126.

[6] Smirnov O, Anselin L. Fast maximum likelihood estimation of very large spatial autoregressive models: A characteristic polynomial approach. Computational Statistics and Data Analysis, 2001, 35: 301-319.

[7] Kelejian H H, Prucha I R. A generalized spatial two-stage least squares procedure for estimating a spatial autoregressive model with sutoregressive disturbance. Journal of Real Estate Finance and Economics, 1998, 17: 99-121.

[8] Kelejian H H, Prucha I R. A generalized moments estimator for the autoregressive parameter in a spatial model. International Economic Review, 1999, 40: 509-533.

[9] Kelejian H H, Prucha I R. Specification and estimation of spatial autoregres-sive models with autoregressive and heteroskedastic disturbances. Journal of Econometrics, 2010, 157: 53-67.

[10] Lee L F. Asymptotic distribution of quasi-maximum likelihood estimators for spatial autoregressive models. Econometrica 2004, 72: 1899-1925.

[11] Su L, Jin S. Profile quasi-maximum likelihood estimation of spatial autoregressive models. Journal of Econometrics, 2010, 157: 18-33.

[12] Lin X, Lee L F. GMM estimation of spatial autoregressive models with unknown heteroskedasticity. Journal of Econometrics, 2010, 157(1): 34-52.

[13] Su L. Semi-parametric GMM estimation of spatial autoregressive models. Journal of Econometrics, 2012, 167: 543-560.

[14] Ai C, Chen X. Efficient estimation of models with conditional moment restrictions containing unknown functions. Econometrica 2003, 71: 1795-1843.

[15] Newey W K. Convergence rates and asymptotic normality for series estimators. Journal of Econometrics, 1997, 79: 147-168.

[16] Lee L F. GMM and 2SLS estimation of mixed regressive, spatial autoregressive models. Journal of Econometrics, 2007, 137: 489-514.

[17] Case A C. Spatial patterns in household demand. Econometrica, 1991, 59: 953-965. 